\title{
The role of nailfold capillaroscopy in monitoring lung involvement in systemic sclerosis
}

\author{
Laura Groseanu ${ }^{1,2}$, Patricia Paraschiva ${ }^{1}$, Andra Balanescu ${ }^{1,2}$, Violeta Bojinca ${ }^{1,2}$, \\ Daniela-Opris Belinski ${ }^{1,2}$, Andreea Borangiu ${ }^{1,2}$, IoanaSaulescu ${ }^{1,2}$, Diana Mazilu ${ }^{1,2}$, \\ Sanziana Daia-Iliescu ${ }^{1,2}$, FlorianBerghea ${ }^{1,2}$, Cosmin Constantinescu ${ }^{1,2}$, \\ Maria-Magdalena Negru' ${ }^{1,2}$, MihaiAbobului ${ }^{1,2}$, ClaudiaCobilinschi ${ }^{1,2}$, Ruxandra Ionescu ${ }^{1,2}$ \\ ${ }^{1 " C a r o l ~ D a v i l a " ~ U n i v e r s i t y ~ o f ~ M e d i c i n e ~ a n d ~ P h a r m a c y, ~ B u c h a r e s t, ~ R o m a n i a ~}$ \\ 2"Sf. Mary" Clinical Hospital, Bucharest, Romania
}

\begin{abstract}
The usefulness of capillaroscopy in the follow-up of scleroderma patients and the possible prognostic role for the appearance of visceral involvement is suggested by many authors but still under debate. The aim of this study was to assess the role of monitoring capillaroscopic abnormalities (qualitative and semiquantitative) in relation with parameters of interstitial lung involvement and pulmonary arterial hypertension(PAH).

A strong correlation was identified between initial capillaroscopy scores and FVC ( $r=-.47, p=0.002)$, DLCO ( $r=-$ $.51, p<0.001)$ and SPAP $(r=0.34, p<0.001)$. Active and late capillaroscopic pattern were correlated with diagnosis of lung fibrosis $(x 2=14, p=0.007)$ and PAH at follow-up examinations $(x 2=14,2, p=0.007)$. Progression of capillaroscopic pattern at follow-up evaluations was not correlated with significant worsening of lung volumes, DLCO, sPAP. Instead, progression of microangiopathy evolution score $(>1)$ was asociated with worsening of FVC $(r=0.32, p<0.001), \operatorname{DLCO}(r=0.21, p=0.02)$ and new diagnosis of lung fibrosis on HRCT $(r=0,19, p=0.035)$.

Semiquantitative scoring, rather then qualitative capillaroscopic assessment can have a predictive role for new involvement or worsening of previous lung involvement (especially interstitial lung disease) in scleroderma patients, confirming the putative role of capillaroscopy as biomarker in SSc.
\end{abstract}

Keywords: nailfold capillaroscopy, lung involvement,monitoring, systemic sclerosis

\section{INTRODUCTION}

Microvascular damage and dysfunction represent the earliest morphological and functional markers of systemic sclerosis(SSc). Nailfold videocapillaroscopy (NVC) is a safe and non-invasive tool to evaluate the morphology of the microcirculation (1). For now, there is no treatment that has proved to halt the natural progression of the clinical recognisable disease. Consequently, eyes are geared to diagnose the disease 'early' before the clinical disease has set in, so effort is being put into the investigation of possible biomarkers. Capillaroscopy is a candidate possible biomarker (2).

In 2000, Cutolo et al classified the progressive microangiopathic changes as assessed by NVC of patients with SSc into the scleroderma patterns (early, active and late) (3). Since 2013 the NVC patterns are included in the European League Against Rheumatism and American College of Rheumatology criteria of SSc (4). The 'Early' SSc pattern is characterized by few enlarged and giant capillaries, few capillary microhaemorrhages, no evident capillary loss and a relatively well preserved capillary distribution. The 'Active' SSc pattern is characterized by frequent giant capillaries, frequent capillary microhaemorrhages, moderate capillary loss, absent or mild ramified capillaries and a mild disorganization of the capillary architecture. In the 'Late' SSc pattern, although giant capillaries and microhaemorrhages are almost absent, there is irregular enlargement of the capillaries, severe capillary loss with extensive avascular areas, ramified or bushy capillaries and a severe disorganization of the capillary array (5). 
The capillaroscopic parameters can also be evaluated by a semi-quantitative scale, consisting of diagnostic parameters (irregularly enlarged capillaries, giant capillaries, micro-haemorrhages) and progression parameters (reduced capillary number, capillary ramifications and capillary architectural disorganization). Score 0-3 is adopted: score 0 : no changes; score $1:<33 \%$ of capillary changes; score $2: 33 \%-66 \%$ of capillary changes; score 3: $>66 \%$ of capillary changes). The mean score value for each parameter is calculated from the analysis of at least two linear millimetres in the middle area of the nailfold bed; the score values from the eight fingers are added together, and the final value divided for eight fingers. The "microangiopathy evolution score" (MES, sum of scores of progression parameters; score 0-9) is used to assess the vascular damage progression, as significantly increase during the evolution of the SSc microangiopathy (6).

NVC analysis allows the detection of microvascular markers of severity and progression in SSc. The „late” scleroderma pattern was significantly associated with a higher risk to develop severe peripheral vascular, skin, joint, muscle, gastrointestinal, lung and heart involvement(6-8). Capillary deletion, severe deformity were correlated with the presence of interstitial lung disease (ILD). The patients with less giant capillaries had more severe ILD involvement. The patients with ILD had significantly higher loss of capillaries score, avascular area score and ramified/bushy capillaries score compared with those without ILD. Moreover, ramified/bushy capillaries score together with diffused SSc were independent risk factors for the presence of ILD (9). Patients with significant loss of capillary density showed worst values of FVC and DLCO(10). The group of Reyes-Reuda also found positive association between abnormal capillaroscopy and interstitial lung disease but no correlation with PAH (11). On the other hand, the group of Ricieri found significant correlations between medium pulmonary arterial pressure (mPAP) values and the NVC score and with the avascular areas score (12).

The purpose of our study was to test on a romanian cohort of SSc patients if more severe NVC abnormalities should lead to strict cardiopulmonary surveillance and a if a complete NVC study is indicated from initial patient evaluation.

\section{METHODS}

We conducted a longitudinal prospective study that included $118 \mathrm{SSc}$ patients monitored between
2013-2017. All patients were diagnosed according to EULAR/ACR 2013 criteria (4). All patients, after giving informed consent, had a demographic,clinical, laboratory and instrumental assessment according to MEDS evaluation sheets (online database for the collection of the Minimal Essential Data Set, the longitudinal anonymized collection of essential clinical data of EUSTAR SSc patients): age, sex, disease duration (defined as Raynaud onset), extend of cutaneous involvement, modified Rodnan skin score (mRSS), digital ulcers/pitting scars, joint/muscle/ gastrointestinal/lung/heart/renal involvement, specific autoantibodies and specific treatment. The organ involvement was defined as previously described (13). Valentini disease activity index was also calculated (14). ILD was diagnosed if any of the following criteria were identified: a) restrictive pulmonary pattern with forced vital capacity (FVC) below $70 \%$ of expected value, b) pulmonary interstitial pattern evidenced by chest radiograph or high-resolution computed tomography scan, or c) alveolitis confirmed by bronchoalveolar lavage (defined as neutrophilia of $\geq 3 \%$, eosinophilia of $\geq 2 \%$, or lymphocytosis $\geq 15 \%$ ). PAH was diagnosed when systolic pulmonary arterial pressure (SPAP) was estimated to be $>35 \mathrm{~mm} \mathrm{Hg}$ by Doppler echocardiogram or when mean PAP was equal or higher than $25 \mathrm{~mm} \mathrm{Hg}$ at rest by right-sided heart catheterization (RHC) (13). Lung involvement worsening was defined as decrease of FVC $>15 \%$, DLCO $>10 \%$, new diagnosis of alveolitis on high resolution computerized tomography or new diagnosis of PAH. PAH worsening was defined as as new diagnosis of PAH or increase of sPAP with $10 \mathrm{mmHg}$. Worsening of MES score was defined as increase of the baseline score with 1 point.

\section{Nailfold videocapillaroscopy}

NVC was performed by a trained rheumatologist using a videocapillaroscope with a X200 magnification probe according to the standard method (3). First, patients were distributed into a proper NVC pattern: early, active, late or nonspecific (3). A semiquantitative rating scale to score the altered microvascular parameters was used as described in the literature (score 0-3) (6). The „microangiopathy evolution score" (MES) was selected to assess the progression of the vascular damage (6).

\section{Statistical analysis}

Student t-test/Mann-Whitney test, chi-square test were used to evaluate differences across subgroups. 
Pearson's bivariate correlation/Spearman's rank correlation coefficient were used to evaluate the association between variables. $P$ values $<0.05$ were considered statistically significant.

\section{RESULTS}

The study group included 118 patients, 103 females $(87,29 \%), 63(53,39 \%)$ had a diffuse extend of skin involvement, mean age was 50.58(12,71) years, mean disease duration at first $\mathrm{NVC}$ evaluation $2,81(4,2)$ years. The whole group had a second evaluation, including a complete NVC study, after a mean interval of 2,26(1,12) years. $17(14,40 \%)$ patients died during the follow-up, 24 (20,33\%) were lost to follow-up. The demographics of the patients are presented in table 1.

TABLE 1. Demographics, clinical and immunological characteristcs of the study group

\begin{tabular}{|l|l|}
\hline & Baseline \\
\hline Number pf patients & 118 \\
\hline Sex ratio F/M & $103 / 15$ \\
\hline Age (years), mean(SD) & $50.58(12,71)$ \\
\hline Disease duration (years), mean(SD) & $2,81(4,3)$ \\
\hline Disease subset dSSc/ISSc & $63 / 55$ \\
\hline Autoantibodies (\%) & \\
antiSCL70+ & 57,63 \\
anticentromer+ & 31,36 \\
both+ & 1,69 \\
\hline moth- & 9,32 \\
\hline Digital ulcers (\%) & $10,02(5,99)$ \\
\hline Musculoarticular involvement (\%) & 41,53 \\
\hline Gastrointestinal involvement (\%) & 45,76 \\
\hline Renal involvement (\%) & 77,96 \\
\hline ILD (\%) & 5,08 \\
\hline PAH (\%) & 33,89 \\
\hline ILD+PAH (\%) & 18,64 \\
\hline FVC (\%predicted), mean(SD) & 9,32 \\
\hline DLCO (\%predicted), mean(SD) & $78,08(14)$ \\
\hline sPAP mmHg, mean(SD) & $65,57(18,69)$ \\
\hline Dyastolic disfunction (\%) & $30,18(10,97)$ \\
\hline Systolic disfunction (\%) & 33,89 \\
\hline Rhythm and condunction disturbances & 10,17 \\
\hline (\%) & 27,11 \\
\hline NVC pattern (\%) & \\
\hline Early & 12,72 \\
Lctive & 46,61 \\
\hline NVC scoring, mean(SD) & 2,54 \\
\hline Ab & $4,76(1,43)$ \\
\hline
\end{tabular}

Abbreviations:

ILD - interstitial lung disease, PAH-pulmonary arterial hypertension, FVC - forced vital capacity, DLCO - diffusing capacity for carbon monoxide, SPAP - systolic pulmonary arterial pressure, NVC - nailfold videocapillaroscopy

\section{Results at baseline evaluation}

$43,22 \%$ of the patients had lung involvement at the first evaluation: $33,89 \%$ had ILD, $18,64 \%$ had PAH. $9,32 \%$ of the patients had both ILD and PAH. $87,5 \%$ of the patients with ILD had a diffuse SSc, 54,54\% among those with PAH had limited form of the disease. $87,5 \%$ of the ILD patients tested positive for antiSCL70 antibodies. 54,54\% among those with PAH tested positive for anticentromer antibodies. Mean FVC was 78,08 (14)\%, mean DLCO was 65,57 $(18,69) \%$, mean sPAP was $30,18(10,97) \mathrm{mmHg}$.

$12,71 \%$ of the patients had an early NVC pattern, $46,61 \%$ had an active pattern and $37,29 \%$ had a late pattern. Mean MES was 4,76 $(1,43)$.

Patients with ILD had higher MES than patiens without ILD [5,58 $(1,35)$ vs 4,35 $(1,28), \mathrm{p}<0,001]$. Patients with $\mathrm{PAH}$ at first evaluation had higher MES than those without PAH $[5,64(1,09)$ vs 4,56 $(1,42), \mathrm{p}<0,001]$.

Active and late pattern were correlated with the diagnosis of ILD [ $\chi 2(4)=9.62, p=0.047]$ and PAH $[\chi 2(4)=15,632, p=0.004]$. Patients with a late capillaroscopic pattern had lower FVC [74,2(15,98)\%)], lower DLCO $[54,75(20,92) \%]$ and higher sPAP $[44(14,83) \%]$. Correlations were identified between baseline MES and FVC ( $\mathrm{r}=-.47, \mathrm{p}=0.002)$, FEV $(\mathrm{r}=-0.45, \mathrm{p}=0.003)$, DLCO $(\mathrm{r}=-0.51, \mathrm{p}<0.001)$ and $\operatorname{sPAP}(\mathrm{r}=0.34, \mathrm{p}<0.001)$.

Figure 1 representation of the correlation between NVC pattern and FVC values $(r=-0,276$, $\mathrm{p}=0,002)(\mathrm{A}), \mathrm{DLCO}$ values $(\mathrm{r}=-0,343, \mathrm{p}<0,001)(\mathrm{B})$ and $\operatorname{sPAP}(\mathrm{r}=0,326, \mathrm{p}<0,001)(\mathrm{C})$.

\section{Results at the follow-up evaluation}

10 more patients $(8,47 \%)$ had new lung involvement at the second evaluation : $42,37 \%$ ILD, $24,42 \%$ PAH; $15,25 \%$ of the patients had both ILD and PAH.

Mean FVC was $73,83(15,9) \%$, the regression rate for patients with ILD was 7,6 $(6,73) \%$; mean DLCO was $60,82(20,35) \%$ and the mean regression rate for patients with ILD was $8(9,15) \%$. Mean sPAP was $32,49(15,79) \mathrm{mmHg}$ with a mean progression of $12,57(15,11) \mathrm{mmHg}$.

Lung involvement (ILD plus PAH) was the main cause of death $(88,23 \%)$.

23 patients had an increase of one step of the NVC pattern, 2 patients with 2 steps. Distribution of the NVC pattern at the second evaluation was as follows: $5,93 \%$ had an early pattern, $38,41 \%$ active pattern and 54,24\% late pattern. Mean MES was 

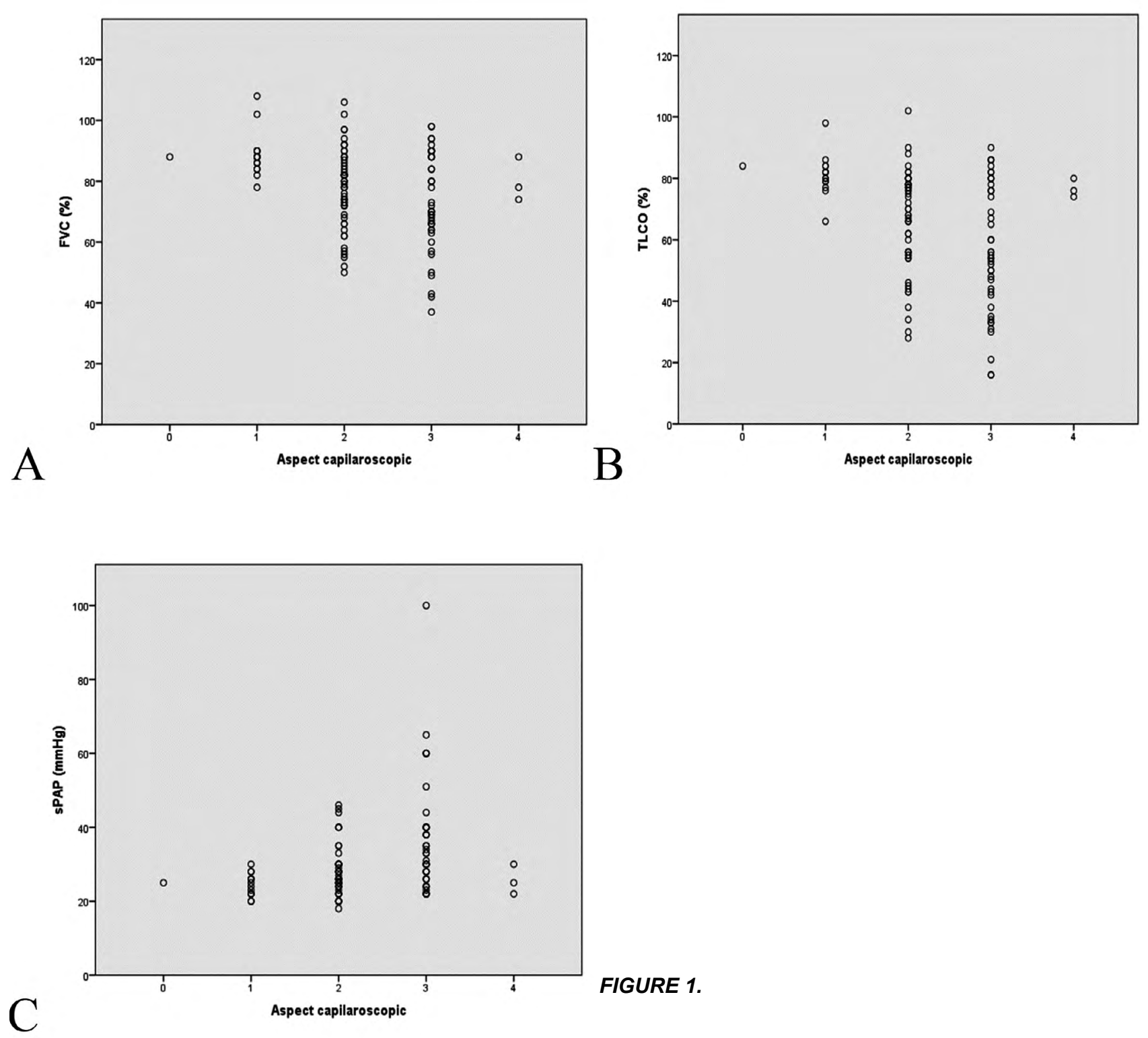

FIGURE 1.

$5,88(1,74)$. The mean increase of MES was $1,19(0,95)$.

Patients with ILD had higher MES scores than those without ILD $[6,68(1,53)$ vs $5,29(1,65)$, $\mathrm{p}<0,001]$. Patients with PAH had higher MES than those without PAH [7(1,5) vs 5,591,65), $\mathrm{p}<0,001]$.

Active and late pattern were correlated with ILD $[\chi 2(4)=14.00, \mathrm{p}=0.007]$ and PAH $[\chi 2(4)=14.20$, $\mathrm{p}=0.007]$. Patients with a late capillaroscopic pattern had lower FVC [68,33 (16,38)\% ], lower DLCO [52,95 (20,31)\%] and higher sPAP [37 $\mathrm{mmHg}$ $(17,00) \%$. A strong correlation was identified between follow-up MES and FVC $(r=-0,592, p<0,01)$, DLCO $(\mathrm{r}=-0,592, \mathrm{p}<0.01)$ and $\operatorname{sPAP}(\mathrm{r}=0,369, \mathrm{p}<0.01)$.

Worsening of the NVC pattern at follow-up was not correlated with lung tests or sPAP. Instead, MES worsening was correlated with ILD worsening: sig- nificant decrease of FVC ( $\mathrm{r}=0.325, \mathrm{p}<0.001)$ or of TLCO $(r=-0.215, p=0,02)$ or new diagnosis of lung fibrosis $(r=0,194, p=0.035)$. No correlations were identify between MES worsening and PAH worsening $(r=0,081, p=0,38)$.

\section{DISCUSSIONS}

NVC is a useful examination for evaluating microvascular changes in the peripheral circulation, thus it has a relevant role for the diagnosis of SS (5). NVC also seems to be helpful in identifying those SSc patients at risk for visceral involvement (7-8). The present study demonstrates an association between baseline and follow-up NVC parameters and severe pulmonary involvement.

Several previous studies found correlations between baseline scleroderma pattern and future se- 
TABLE 2. Nailfold capillaroscopy characteristics of patients with interstitial lung disease and pulmonary hypertension

\begin{tabular}{|l|l|c|c|c|c|c|}
\hline & & $\begin{array}{c}\text { \% of study } \\
\text { group }\end{array}$ & $\begin{array}{c}\text { Early } \\
\text { pattern }\end{array}$ & $\begin{array}{c}\text { Active } \\
\text { pattern }\end{array}$ & $\begin{array}{c}\text { Late } \\
\text { pattern }\end{array}$ & MES \\
\hline ILD & baseline & 33,89 & 12,17 & 46,61 & 37,29 & $5,58(1,35)$ \\
\hline & follow up & 42,37 & 0,47 & 4,7 & 76,59 & $6,68(1,53)$ \\
\hline PAH & baseline & 18,64 & 5,93 & 38,41 & 54,24 & $5,64(1,09)$ \\
\hline & follow-up & 24,42 & 0 & 17,24 & 82,75 & $7(1,5)$ \\
\hline
\end{tabular}

ILD - interstitial lung disease, PAH-oulmonary hypertension, MES-microangiopathic evolution score

TABLE 3. Characteristics of lung function tests and pulmonary arterial pressure according to nailfold capillaroscopy pattern

\begin{tabular}{|l|l|c|c|c|c|}
\hline & & Early pattern & Active pattern & Late pattern & P value \\
\hline FVC, mean(SD) \%predicted & baseline & $88,8(7,43)$ & $77,96(12,67)$ & $74,2(15,98)$ & 0,01 \\
\hline & follow-up & $90(8,64)$ & $78,56(12,68)$ & $68,33(16,38)$ & $<0,01$ \\
\hline DLCO, mean(SD) \%predicted & baseline & $81,13(6,65)$ & $66,87(16,15)$ & $57,4(20,92)$ & $<0,01$ \\
\hline & follow-up & $84,57(6,39)$ & $67,38(16,09)$ & $52,95(20,31)$ & $<0,01$ \\
\hline sPAP, mean(SD) $\mathbf{m m H g}$ & baseline & $24,27(3,03)$ & $27,73(6,39)$ & $44(14,83)$ & 0,01 \\
\hline & follow-up & $22,57(1,51)$ & $27,84(13,26)$ & $47(17,23)$ & $<0,01$ \\
\hline
\end{tabular}

FVC - forced vital capacity, FVC - forced vital capacity, DLCO - diffusing capacity for carbon monoxide, SPAP - systolic pulmonary arterial pressure

vere lung involvement. The odds rise according to worsening scleroderma patterns. The group of $\mathrm{V}$. Smith in a single/multiple regression analysis found that the odds to develop future severe lung disease were $2.54 / 2.33$ for the early versus the normal pattern, $6.43 / 5.44$ for the active versus the normal pattern and 16.30/12.68 for the late versus the normal pattern. In particular, the most severe microangiopathy pattern on capillaroscopy, namely the late scleroderma pattern, had the highest risk: 16.07 for future severe peripheral vascular disease and 12.68 for future severe lung disease (7).

In our cohort, the number of patients having severe pulmonary involvement significantly differed according to baseline NVC pattern. None of patients with normal baseline NVC pattern, while $2,5 \% / 52,5 \% / 45 \%$ of patients with early/active/late scleroderma pattern had lung involvement. Also, the number of patients having future severe lung involvement significantly differed according to baseline NVC patterns: $0 \%$ of patients with normal baseline NVC pattern, while $0 \% / 20 \% / 80 \%$ with early/ active/late scleroderma pattern had future severe lung disease. Patients with active and late pattern had significantly lower FVC and DLCO volumes compared to early and normal pattern (Table 2, 3). Moreover, the late capillaroscopic pattern and higher MES are related to lower percentage FVC, DLCO and SPAP values independently of other baseline or follow-up characteristics. Worsening of the capil- laroscopic pattern at the follow-up evaluation was not correlated in our study with lung tests (FVC or DLCO). Instead, MES worsening was correlated with significant decrease of FVC or of TLCO or new diagnosis of lung fibrosis. Prior publications have found correlations between baseline specific quantitative NVC studies and lung function test or sPAP. The group of Guillén-Del-Castillo identified that the number of capillaries with neoangiogenesis, male gender and the presence of ILD on HRCT were factors independently associated with lower FVC values (15). SSc patients with ILD had a lower capillary density and a higher number of capillaries with neoangiogenesis. Avouac et al. also identified higher mean avascular scores in patients with ground-glass opacities (16). A progressive loss of capillaries from baseline predicted overall disease progression, new DU, lung vascular progression defined as new onset of precapillary PAH, skin fibrosis and worsening in the Medsger severity score. Similarly, Castellvi et al. (10) described an association between low capillary density and lower FVC and DLCO percentages using a semi-quantitative NVC.

In our study, correlations were also identified between baseline and follow up late NVC pattern and PAH (Table 2, 3). Among patients with PAH at baseline $72,72 \%$ a late pattern, all patients with $\mathrm{PAH}$ worsening at follow-up had a late NVC pattern. Active and late capillaroscopic pattern and MES were 
related to SPAP value at baseline and follow-up. But we could not identify an association between MES worsening and PAH worsening. These might be related to some limitations of the study. In our cohort, the prevalence of PAH was $18.64 \%$ at baseline, a little bit higher then previous reports, related to longer disease duration until the patients were first evaluated in our reference center. However, PAH was estimated by indirect measurements, in few patients was confirmed by RHC.

Hofstee et al. found that capillary density was inversely correlated with the mPAP in both SSc-PAH and idiopathic PAH patients (17). Interestingly, Riccieri (12) demonstrated that higher NVC scores and avascular areas scores were correlated with mPAP. Corrado et al.(18) observed a reduced capillary density and an increased mean capillary width and mean number of capillaries with neoangiogenesis in SSc-
PAH patients compared to SSc patients without PAH evidence.

\section{CONCLUSIONS}

Active and late capillaroscopic pattern are associated with increased frequency of interstitial lung disease and pulmonary hypertension in SSc patients. Semiquantitative scoring (microangiopathy evolution score), rather then qualitative capillaroscopic assessment can have a predictive role for new or worsening of previous lung involvement (especially interstitial lung disease) in SSc patients, confirming the putative role of capillaroscopy as biomarker in SSc. In our opinion, a correct approach to SSc patients should always include a complete NVC study in order to identify early those cases who may possibly develop severe complications such as ILD and PAH.

Conflict of interest: none declared Financial support: none declared

\section{REFERENCES}

1. Smith $V$, Thevissen $K$, Trombetta AC, Pizzorni C, Ruaro B, Piette Y, Paolino S, De Keyser F, Sulli A, Melsens K, Cutolo M; EULAR Study Group on Microcirculation in Rheumatic Diseases.- Nailfold Capillaroscopy and Clinical Applications in Systemic Sclerosis. Microcirculation. 2016; 23(5):364-72.

2. Colmenares Roldána LM, Velásquez Franco CJ, Mesa Navas MA. Capillaroscopy in systemic sclerosis: Revista Colombiana de Reumatologia A narrative literature review 2016;23(4):227-276

3. Cutolo M, Pizzorni C, Secchi ME, Sulli A. Capillaroscopy Best Pract Res Clin Rheumatol. 2008; 22(6):1093-108

4. van den Hoogen F, Khanna D, Fransen J, Johnson SR, Baron M, Tyndall A, Matucci-Cerinic M et al , 2013 classification criteria for systemic sclerosis: an American College of Rheumatology/ European League against Rheumatism collaborative initiative. Arthritis Rheum. $2013 ; 65(11): 2737-47$

5. Cutolo M, Smith V. State of the art on nailfold capillaroscopy: a reliable diagnostic tool and putative biomarker in rheumatology?, Rheumatology, 2013;52(11): 1933-1940

6. Sulli A, Secchi ME, Pizzorni C, Cutolo M. Scoring the nailfold microvascular changes during the capillaroscopic analysis in systemic sclerosis patients. Ann Rheum Dis. 2008 ;67(6):885-7

7. Smith $V$, Cutolo M. Do worsening scleroderma capillaroscopic patterns predict future severe organ involvement? a pilot study. Ann Rheum Dis. 2012;71(10):1636-9

8. Cutolo M, Smith V. State of the art on nailfold capillaroscopy: a reliable diagnostic tool and putative biomarker in rheumatology? Rheumatology (Oxford) (2013) 52 (11): 1933-1940.

9. Ji LL, Wang H, Zhang XH, Zhang BL. Correlation between nailfold capillaroscopic findings and presence of interstitial lung disease in systemic sclerosis patients. Beijing Da Xue Xue Bao Yi Xue Ban 2018 Jun 18;50(3):501-506.

10. Castellví I, Simeón-Aznar CP, Sarmiento M, Fortuna A, Mayos $M$, Geli C Nailfold capillaroscopy findings are related to the pulmonary function tests values in patients with systemic sclerosis J Rheumatol. 2015;42(2):222-7.

11. Reyes-Rueda J, Gutierrez-Ureña S, Gonzalez-Díaz V et al. AB0771 Abnormal capillaroscopy and pulmonary hypertension in patients with systemic sclerosis Annals of the Rheumatic Diseases 2018;77:1520.

12. Riccieri V, Vasile M, lannace N, Stefanantoni K, Sciarra I, Vizza $\mathrm{CD}$ et al. Systemic sclerosis patients with and without pulmonary arterial hypertension: a nailfold capillaroscopy study Rheumatology (Oxford). 2013 ;52(8):1525-8.

13. Alba MA, Velasco C, Simeón CP et al. Early- versus late-onset systemic sclerosis: differences in clinical presentation and outcome in 1037 patients. Medicine (Baltimore). 2014;93(2):73-81.

14. Valentini G, Bencivelli W, Bombardieri S, D'Angelo S, Della Rossa A, Silman AJ et al. European Scleroderma Study Group to define disease activity criteria for systemic sclerosis. III. Assessment of the construct validity of the preliminary activity criteria. Ann Rheum Dis. $2003 ; 62(9): 901-3$

15. Guillén-Del-Castillo A, Simeón-Aznar CP, Callejas-Moraga EL et al. Quantitative videocapillaroscopy correlates with functional respiratory parameters: a clue for vasculopathy as a pathogenic mechanism for lung injury in systemic sclerosis. Arthritis Res Ther. 2018;20(1):281.

16. Avouac J, Lepri G, Smith V, Toniolo E, Hurabielle C, Vallet A et al. Sequential nailfold videocapillaroscopy examinations have responsiveness to detect organ progression in systemic sclerosis. Semin Arthritis Rheum. 2017;47:86-94.

17. Hofstee HM, Vonk Noordegraaf A, Voskuyl AE, Dijkmans BA, Postmus PE, Smulders YM et al. Nailfold capillary density is associated with the presence and severity of pulmonary arterial hypertension in systemic sclerosis. Ann Rheum Dis. 2009; 68:191-195

18. Corrado A, Correale M, Mansueto N, Monaco I, Carriero A, Mele A et al. Nailfold capillaroscopic changes in patients with idiopathic pulmonary arterial hypertension and systemic sclerosis-related pulmonary arterial hypertension. Microvasc Res. 2017;114:46-51 